\title{
Fractal dimension of solar wind high speed flows
}

\section{T. E. Val'chuk}

IZMIRAN, 142190, Troitsk, Moscow Region, Russia email: valchuk@izmiran.troitsk.ru

The investigation of coronal holes $(\mathrm{CH})$ is in progress now thanks to a number of high precise experimental projects and a lot of observations (Ulysses, SOHO, Yohkoh and many others). Historical epoch of $\mathrm{CH}$ discovery and study is prolonged today on the new experimental materials. Our considerations of $\mathrm{CH}$ and its manifestations in SW near the Earth were connected with minimum, growth and maximum phases of solar cycle. In this work we deal with the descending phase of solar cycle 23. There was the interval of comparatively quite Sun in March 2004, when strong sporadic phenomena were not observed. The existence of long-lived transequatorial recurrent coronal holes on the solar disk allows the investigation it imprinting in SW flows, using Wind data in near Earth cosmic space, data SOHO, Yohkoh and others.

The inhomogeneous and complex solar magnetosphere and a number of heliomagnetic phenomena are reflected in solar wind (SW) parameters. There are no simple connections, but the extraction of phenomena in equatorial solar region allows fractal characteristic analysis of SW parameters, which are corresponding with these long-lived phenomena. The open solar magnetosphere and SW streams are investigated from the point of view of the coronal hole manifestations - on the Sun and in SW near the Earth. Fractal dimension (FD) calculations was performed by Higuchi method, FD was calculated for high speed SW flows from positive and negative $\mathrm{CH}$ in March 2004, as well as in the boundary and background regions. FD values of all SW plasma parameters in the region of $\mathrm{CH}$ high speed flows in SW are about 1.8-1.9. It is typical for manifestations of high-speed flows from $\mathrm{CH}$. These calculations confirm the fractal nature of SW and give the opportunity to evaluate the fluctuation characteristics of different regions.

SW high speed streams from $\mathrm{CH}$ reveal a similar FD characteristics and analogous features in SW in general. Differences are accounted for by the level of solar disturbance near the $\mathrm{CH}$ location. The transition of the heliospheric current layer (twice in March 9 and 24) reveals the deepest fall of the fractal dimensions. Heliosphere current layer has a different fractal structure as evidenced by a sharp decrease of the fractal dimension.

The fractal nature of the solar and SW plasma is discussed; the Sin-Gordon equation

$$
\frac{\partial^{2} B}{\partial t^{2}}-C_{S}^{2} \frac{\partial^{2} B}{\partial x^{2}}+\omega_{0}^{2} \sin v=0,
$$

where $B$ - magnetic field, $C_{s}$ - sound velocity, $\omega_{0}$ - oscillation frequency of "net", $v$ angle of direction of generated soliton flow, has soliton decision of kink type and a chain of discrete MGD elements, and it's solution (Ryskin et al. (2000))

$$
B=4 \arctan \exp \left( \pm \frac{\omega_{0}}{C_{S}} \xi\right),
$$

( $\xi$ - current radial co-ordinate) are proposed as a probable approach to the analytical consideration this problem. New $\sim 1 \operatorname{arcsec}^{2}$ resolution observations (Lin et al. (1999)) (high precision infrared polarimetry and high-resolution imagery in the visible) show magnetic features. Their magnetic field strengths range from below 200 to $10^{3}$ Gs. The 
evolution of these magnetic features are associated with the solar granulation, the most interesting process is the formation and disappearance of a kilogauss magnetic features in inter-granular lanes. Strong magnetic field features are concentrated in the small isolated areas with an average filling factor of the order of $1 \%$ on solar disk. Observations at 0.1 " resolution of the dynamic evolution of magnetic elements are corresponded by M.Carlsson et al., 2004, (in this issue).

It is known, that oscillations of the granular network on the transition region heights (chromosphere - corona) are intensified especially effectively in unipolar magnetic field of $\mathrm{CH}$, the region of high speed SW stream generation. It will be possible the generation of MGD solitons in the medium with the dispersion in the low frequency band.

The generation of nonlinear Alfven waves on the fractal magnetic net of photospheric granulation is discussed as a possible model of the high velocity flows origination (Almeida (2001), Banerjee et al. (2001)). The flow similarity from the $\mathrm{CH}$ recurrent regions and boundary region features have the explanation in the frames of this connection.

The quality model of high speed flows of SW from $\mathrm{CH}$ has following characteristics: the kink (in parameters of magnetic field and plasma), connected with structure and boundaries of $\mathrm{CH}$, defines overall restricted region of high speed flows, this region is fill up streaming of magneto- plasma elements with high velocity and low plasma density. The flow of discrete chains is generated above unipolar magnetic field area of $\mathrm{CH}$ only.

The fractal dimension calculations (Higuchi (1988)) of SW IMF and plasma CH parameters confirm the high medium turbulence and reveal the discrete soliton chains also. The kink solution is similar to bow shock, but has all characteristics of soliton.

CONCLUSION: FD calculations help us to understand SW flow structure and to identify of high speed SW streams from coronal holes.

The front boundary zone of high speed SW is characterized with growth of proton density $\mathrm{N}$ and magnetic clouds in the interplanetary magnetic field. The velocity of SW only begins own increase in this time interval. Than SW velocity reaches of a maximal values and parameter $\mathrm{N}$ is diminished up to several units. Such picture may exist in the absence of sporadic phenomena (flares, filament explosions, coronal mass ejections and others).

All these characteristics of SW parameters from $\mathrm{CH}$ flows show the similarity in all phases of solar cycle, if only mentioned above condition of quite Sun is realized in the interval of SW research.

The transition of heliospheric current layer (twice in March - 9 and 24) in SW flows is elucidated in the deepest fall of fractal dimension values, $\mathrm{D} \sim 1.5$. Heliospheric current layer has another fractal structure as evidenced by the sharp decrease of fractal dimension.

The quality model of stationary high speed flow of SW from $\mathrm{CH}$, including the flux of Alfven solitons, generating of magnetic chromosphere network, is proposed.

Author is grateful to Yohkoh, SOHO/EIT and Wind teams, as well as V.N.Ishkov, E.I.Mogilevsky and K.I.Nikolskaya. This work is supported by RFFI GR 040216374a.

\section{References}

Almeida, J. S. 2001 Astrophys. J. 556, 928-936.

Banerjee D., O'Shea E., Doyle J.G., \& Faurobert M. 2001 Astronomy and Astrophys. J.371, 1123-1149.

Higuchi T. 1988 Physica D31, 277-283.

Lin H., Rimmele T. 1999 Astrophys. J. 514, 448-455.

Riskin, N.M., Trubezkov, D.I. 2000 Nonlinear waves. Moscow, Nauka-Fizmatlit, 268p. 